\title{
DRA BAR-PULL CONTROL SYSTEM OF A FLEXIBLE TRACKED VEHICLE ON WEAK TERRAIN
}

Tatsuro MURO, Prof. Department of 0cean Engineering Faculty of Engineering, Ehi 1 e University

3 Bunkyo-cho, Matsuyara, Japan

\section{ABSTRACT}

To establish a dramar-pull control system to obtain a maximum productivity of a flexible tracked vehicle i.e. a bulldozer running on a super weak marine sediment, the traffic performance of vehicle could be clarified by use of a micro-computer in robotics for construction from the initial information of terrain properties and vehicle dimensions. The optimum dravbar-pull at an optimum slip ratio in dozing operation of an actual large bulldozer running on a self-consolidated kaolinite clay sediment could be determined from the relation between driving force, drawbar-pull, sinkage, trim angle and slip ratio. And also, the tractive performance at braking state i.e. the relations between braking force, effective braking force, sinkage and slip ratio could be presented.

\section{Introduction}

As an underwater vehicle runing on a super veak marine sediment, it should have flexible rubber tracks and larger front and rear wheel in order to accomodate rubber tires of sufficient durability and to provide enough suspension. As the terrain properties, cohesion, angle of internal friction, modulus of shear deformation to calculate the thrust of vehicle, and pressure sinkage curve at rest and relations between slip sinkage, contact pressure and arount of slippage to calculate the locomotion resistance could be estimated by use of input data at several slip ratios from some sensors wich could measure the sinkage of rear sprocket at initial and slippage state, the trim angle and the torque.

2. Flexible tracked vehicle

Fig. 1 shows a part of the flexible rubber track belt, of which road wheel spacing $R_{p}$ is 100 cri and grouser pitch $G_{p}$ is $23 \mathrm{~cm}$. For flexible tracks, the pressure distribution along the track belt becomes nonuniform. The magnitude of peak pressure under the road wheels depends not only on the number of road wheels and the track tension but also on the soil properties ${ }^{\text {s }}$.

Here, assuming that the peak pressure becomes to be 1.2 times as large as the contact pressure acting on a rigid

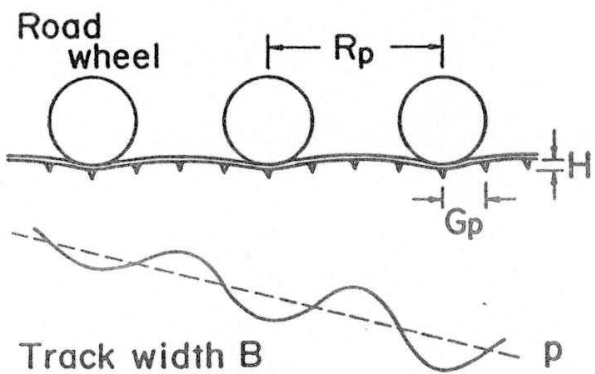

Fig. 1 Pressure distribution alog flexible rubber track belt.

track belt, the multipeak sinusoidal pressure distribution described in Eq. (1) is supposed to act under the flexible tracks.

$$
\left.p=\left\{p_{r}+\left(p_{r}-p_{r}\right) \frac{X}{D}\right\}\left\{1+\frac{1}{5} \cos 2 \pi n \frac{X}{D}\right)\right\}
$$

where $p$ is the contact pressure at the distance $X$ from the frontidler, $p_{r}$ and $p_{r}$ is the pressure under the botton of front and rear whel respectively, D is the contact length of track belt, and $n$ is the number 


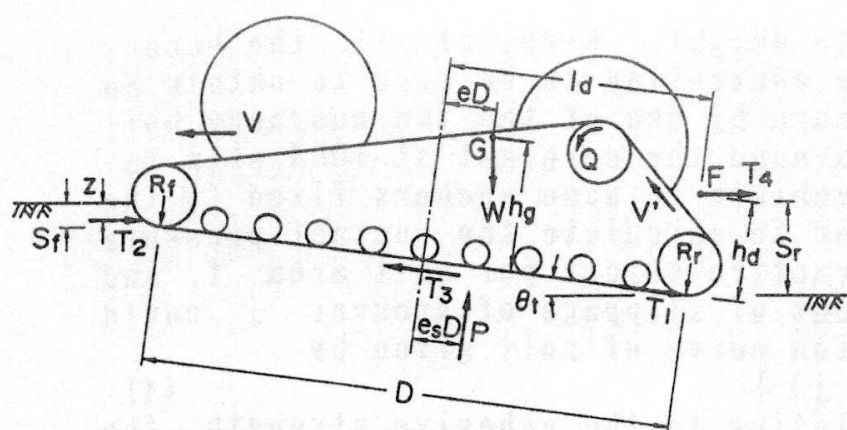

Fig. 2 Flexible tracked undervater vehicle; Dimensions and several forces.

of periods.

Fig. 2 shows the dimensions of underwater vehicle having two buoyancy tanks to control the contact pressure and the sinkage of track belt. To decrease the locomotion resistance due to an excessive slip sinkage, the contact pressure described in Eq. (2) could be decreased by controlling the vehicle weight and the eccentricity of gravity center e by use of the right and left buoyancy tanks.

$p_{F}=(1-6$ e $) \cos \theta_{t} / 2 B D$

$p_{r}=(1+6$ e $) \cos \theta_{t} / 2 B$ D

where $B$ is the idth of track belt and $\theta_{\text {t }}$ is the trim angle.

Here, the dimensions of underwa-

ter flexible tracked rehicle shown in Table 1 are used to analyze the tractive performance at driving and braking state.

\section{Terrain properties}

To determine the static sinkage of track belt, the relations between contact pressure $p_{f}, p_{r}$ and the corresponding sinkage $S_{\mathrm{f}}, S_{r}$ should be measured in-situ automatically for various vehicle weight during the settlement of vehicle on the terrain surface. Load cell sensor to measure sors to measure $S_{T}$ and $\theta_{t}$ could be used to decide the static pressure sinkage curve given in Eq. (3).

$$
S=b_{0} p^{\text {in }}
$$

The constants $b_{0}$ and $b_{1}$ are shown in Table 2 for the marine sediment of kaolinite clay ${ }^{2)}$ self-consolidated under sea water.

To determine the tractive effort, i.e. the thrust or the drag force wich is developed on the interface between track belt and marine sediment, the relation between tractive effort, contact pressure and amount of slippage should be
Table 1 Dimensions of underwater vehicle

\begin{tabular}{|c|c|c|}
\hline $\begin{array}{l}\text { Veight of vehicle at } \\
\text { submerged state }\end{array}$ & $\|$ & $44.1 \mathrm{kN}$ \\
\hline Width of track belt & B & $200 \mathrm{ca}$ \\
\hline $\begin{array}{l}\text { Contact length of } \\
\text { track beit }\end{array}$ & B & $1000 \mathrm{~cm}$ \\
\hline $\begin{array}{l}\text { Mean contact } \\
\text { pressure }\end{array}$ & $p_{m}$ & $1.10 \mathrm{kPa}$ \\
\hline Radius of frontidler & $\mathbf{R}_{\mathbf{f}}$ & $60 \mathrm{c}$ \\
\hline Radius of rear wheel & $\mathbf{R}_{\mathbf{r}}$ & $80 \mathrm{~cm}$ \\
\hline Height of grouser & H & $4.5 \mathrm{~cm}$ \\
\hline Grouser pitch & $\mathbf{G}_{\mathbf{p}}$ & $23 \mathrm{~cm}$ \\
\hline Road wheel spacing & $\mathbf{R}_{\mathbf{p}}$ & $100 \mathrm{cta}$ \\
\hline Eccentricity & e & 0.00 \\
\hline $\begin{array}{l}\text { Height of gravity } \\
\text { center of vehicle }\end{array}$ & $\mathbf{h}_{\mathbf{m}}$ & $250 \mathrm{~cm}$ \\
\hline $\begin{array}{l}\text { Distance betreen } \\
\text { central axis of } \\
\text { rehicle and point } \\
\text { acting drabar-pull }\end{array}$ & Id & $500 \mathrm{c}$ \\
\hline $\begin{array}{l}\text { Height of point } \\
\text { acting drawbar-pull }\end{array}$ & $\mathbf{h}_{\mathbf{d}}$ & $100 \mathrm{~cm}$ \\
\hline $\begin{array}{l}\text { Speed of track belt } \\
\text { against rehicle }\end{array}$ & $v^{\prime}$ & $100 \mathrm{~cm} / \mathrm{s}$ \\
\hline
\end{tabular}

\section{Table 2 Soil properties}

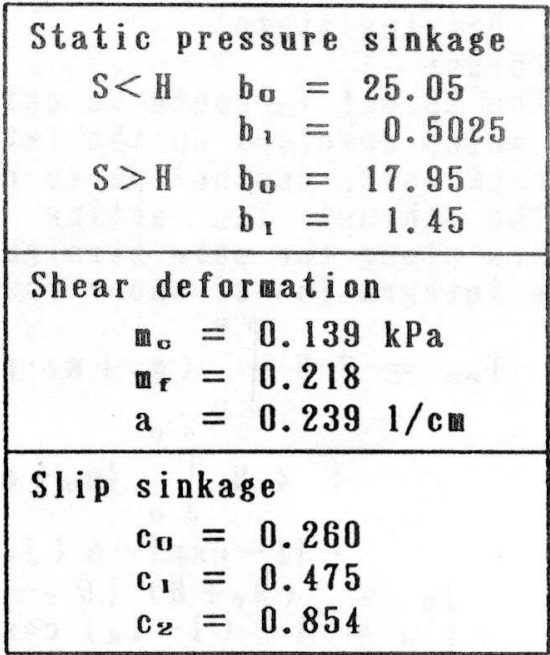


measured in-situ for various vehicle reight. First of all, the eccentricity of gravity center e should be controlled to be zero to obtain an unifor distribution of contact pressure by use of the two buoyancy balancers. Next, drive the track belt around the sprocket at $100 \%$ slip ratio after connecting the undervater vehicle to some anchors fixed to the botton of the sea. Load cell sensor to calculate the contact pressure $p$, torque sensor to calculate the tractive effort per unit area $f$, and rotation sensor to calculate the amount of slippage of grouser $j$ could be used to decide the shear deformation curve of soil given by

$$
f=\left(m_{0}+m_{\mathbf{f}} \cdot p\right)\{1-\exp (-\mathbf{a} j)\}
$$

where wo, $^{-1}$ and a are constants relating to the cohesive strength, the angle of internal friction, and the modulus of deformation of soila).

Those constants are shown in Table 2 for the same marine sediment.

To determine the slip sinkage of rear weel given as the sumation of each value along the track belt under various contact pressure and amount of slippage, the relation between them should be measured in-situ at the same time. On the same process as the shear deformation curve measurement, the slip sinkage of the grouser takes the same value along the track belt because of the same amount of slippage at any position and it increases ith the increment of the travelling time. From the relations between the slip sinkage of track belt $S_{s}$ and the amount of slippage $j$ i.e. the product of travelling time and the speed of track belt for various contact pressure p, the following equation could be derived as

$$
S_{s}=c_{0} p^{\mathbf{1}} j^{02}
$$

where $c_{0}, c_{1}$ and $c_{2}$ are constants relating to grouser type and shear strength of soil. Also, those constants for the same terrain are shon in Table 2 .

\section{Simulation programe}

\subsection{Tractive effort}

As shown in Fig. 2, the effective driving or braking force $T_{4}$ could be determined from the force balances between driving or braking force $T_{1}$, compaction resistance $T_{2}$, thrust or drag force $T_{3}$, resultant normal force $P$ and as

$$
\mathrm{T}_{4}=\frac{\mathrm{T}_{3}}{\cos \theta_{t}}-\tan \theta_{t}-\mathrm{T}_{2}
$$

where $\theta_{t}$ is trim angle

4.1.1 Driving state

(a) Thrust

The thrust $T_{3}$ could be calculated as the sumation of shear resistance which develops on the interface between main straight part of bottom track belt, contact parts of front and rear weel and terrain.

The thrust $T_{m b}$ acting on the bottor and the 4 side parts of grousers along the rain straight part of botton track belt is calculated as the integration of shear resistance 4.5$)$.

$$
\begin{aligned}
& T_{m b}=2 B \int_{0}^{D}\left(m_{B}+w_{r} \cdot p\right)\left[1-\exp \left\{-a\left(j_{B}+i^{\prime} d X\right)\right\}\right] d X \\
& +4 H \int_{0}^{D}\left\{m_{0}+m_{f} \frac{p}{\pi} \cot ^{-1}\left(\frac{H}{B}\right)\right\} \\
& \text { - }\left[1-\exp \left\{-\mathbf{a}\left(j_{B}+i^{\prime} a X\right)\right\}\right] d X \\
& j_{B}=\left(R_{F}+H\right)\left[\theta_{F}-\left(1-i_{a}\right)\left\{\sin \left(\theta_{F}+\theta_{t}\right)-\sin \theta_{t}\right\}\right] \\
& i^{\prime}=1-\left(1-i_{d}\right) \cos \theta_{t} \\
& i_{d}{ }^{d}=1-v / v \text {, }
\end{aligned}
$$


and $\theta_{F}$ is the entry angle, $i_{a}$ is the slip ratio, $V$ is the horizontal vehicle speed and $V^{\text {P }}$ is the rotation speed of track belt at the top of grousers. The thrusts $T_{\text {fd }}$ and $T_{r d}$ developed on the contact parts of front and rear whel could also be calculated as the integration of shear resistance acting on the bottoris and the 4 sides of grousers in the direction of the main straight part of track belt.

Therefore, the thrust $T_{3}$ is given by

$T_{3}=T_{m a}+T_{r d}+T_{r d}$

where $T_{r a}$ is zero in the case of the eccentricity es larger than 1/6.

(b) Compaction resistance

The vertical sinkage $S_{r}$ is calculated as the sumation of static sinkage given from $b_{0}\left(1.2 \cdot p_{r}\right)^{b 1} \cos \theta_{t}$ and slip sinkage $S_{r i}$. The rinute slip sinkage $\Delta S_{i}$ during the inute interval d could be given as

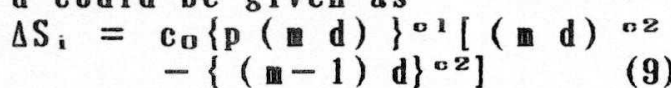

$$
\begin{aligned}
& p(\text { 面 } d)=\left\{p_{\mathrm{p}}+\left(p_{\mathrm{r}}-\mathrm{p}_{\mathrm{P}}\right)\right. \\
& \text { - } \left.\left(\frac{1-1 / 2}{M}\right)\right\}\left\{1+\frac{1}{5}\right. \\
& \left.\cdot \cos \left(20 \pi \frac{m-1 / 2}{M}\right)\right\} \\
& d=\frac{1}{M} \cdot \frac{i^{\prime} d}{1-i^{\prime} d} \quad e_{s} \leqq \frac{1}{6}
\end{aligned}
$$

Md is the total amount of slippage i.e. the shear deformation of soil on the terrain surface during the passage of vehicle $e^{(3)}$.

Then the vertical slip sinkage at rear weel is given by integrating the above equation as

$$
S_{r i}=\left(\sum_{m=-1}^{M} \Delta S_{i}\right) \cdot \cos \theta_{t}
$$

Therefore, $T_{2}$ is calculated by

$$
T_{2}=\frac{2 b_{1} B}{1+b_{1}}\left(\frac{1}{b_{0}}\right)^{1} 1 / b_{1}
$$

(c) Drawbar-puli

Fig. 3 shows the flow chart for calculating the traffic performance of flexible tracked rehicle runing on the superweak marine sediment. First of all, the initial data: $\quad B, D, R_{r}$, $R_{r}, p_{m}, H, e, h_{g}, l_{d,}, h_{d}$ and $V$ 'are giren. And the soil constants bo, $b_{1}$, 嘈o,

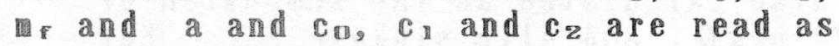
input data. At rest, $p_{r}, p_{r}$ and $S_{r}$, $S_{r}$, and $\theta_{t}$ are repeatedly calculated until the eccentricity eso is determined. At slippage state for a given id, pri, pri, $S_{r}=S_{r o}, S_{r}=S_{r a}+S_{r i}$, $\theta_{\text {ti, }} \mathrm{T}_{3}, \mathrm{~T}_{2}, \mathrm{~T}_{4}$ are repeatedly calculated until the eccentricity $e_{s}$ is determined. For each slip ratio id from zero to 100\%, $T_{1}$ and $T_{4}$ could be calculated by use of a microcomputer and the results are shown in Fig. 4 .

$T_{1}$ decreases gradually with id

from the raximum value $16.8 \mathrm{kN}$ at $i_{d}=2 \%$. Also $\mathrm{T}_{4}$ decreases rapidly with $\mathrm{i}_{\mathrm{d}}$

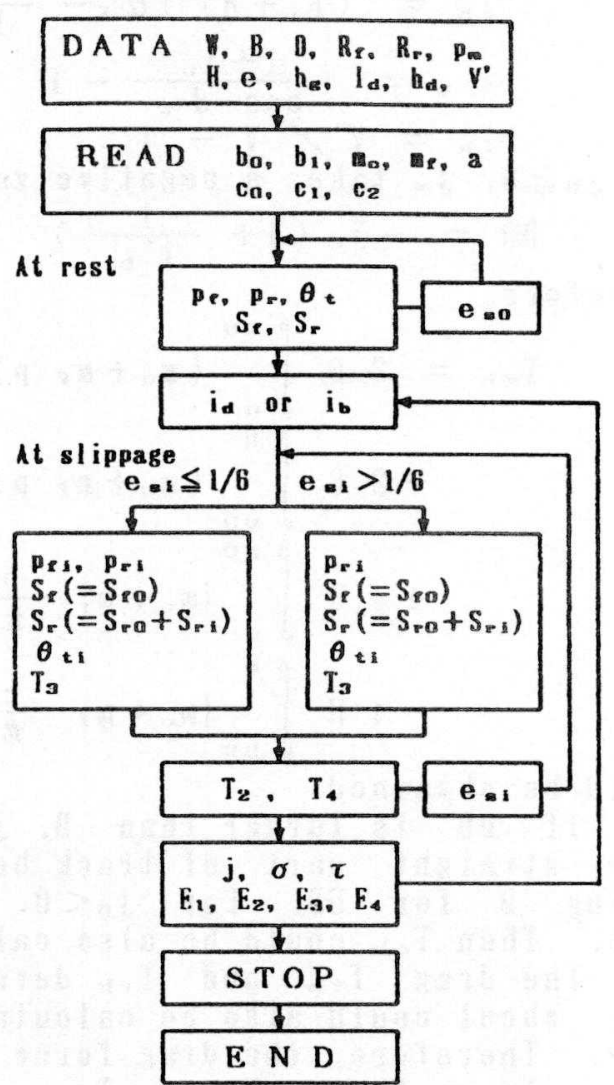

Fig. 3 Flow chart

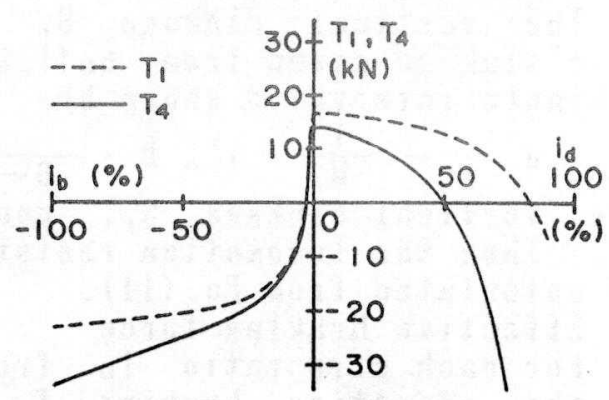

Fig. 4 Relations between driving (or braking) force $T_{1}$, drawbar-pull (or effective braking force) $\mathrm{T}_{4}$ and slip ratio i. 
from the raximum one $14.2 \mathrm{kN}$ at $i_{\mathrm{a}}=1 \%$ due to the increase of compaction resistance.

\subsubsection{Braking state}

(a) Drag force

The thrust $T_{m b}$ acting on the botton and the 4 side parts of grousers along. the main straight part of track belt is also calculated for both positive and negative amount of slippage as the integration of shear resistance. The amount of slippage $j_{\text {m }}$ is given by

$$
\begin{aligned}
j_{m} & =\frac{i_{b}^{\prime}}{1+i_{b}} x+j_{B} \\
j_{B} & =\left(R_{F}+H\right)\left[\theta_{r}-\frac{1}{1+i_{b}}\left\{\sin \left(\theta_{F}+\theta_{t}\right)-\sin \theta_{t}\right\}\right] \\
i_{b} & =\frac{1+i_{b}}{\cos \theta_{t}}-1 \\
i_{b} & =V / V-1
\end{aligned}
$$

For $j_{в} \geqq 0, j_{m}$ takes a negative value from $X=D D$ expressed as

$$
\mathrm{DD}=-\mathrm{j}_{\mathrm{B}}\left(1+\frac{1}{\mathrm{i}_{\mathrm{b}}^{\mathrm{b}}}\right) \text {. }
$$

Therefore,

$$
\begin{aligned}
& \mathrm{T}_{\mathbf{m} \boldsymbol{b}}=2 \mathrm{~B} \int_{\mathbf{D}}^{\mathrm{DD}}\left(\mathbf{m}_{\mathbf{c}}+\mathbf{m}_{\mathbf{r}} \mathbf{p}\right)\left\{1-\exp \left(-\mathbf{a} \mathrm{j}_{\mathbf{m}}\right)\right\} \mathrm{dX} \\
& -2 B \int_{D D}^{D}\left(m_{B}+w_{P} p\right)\left\{1-\exp \left(a j_{m}\right)\right\} d X
\end{aligned}
$$

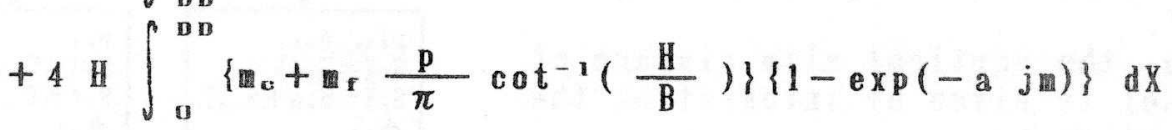

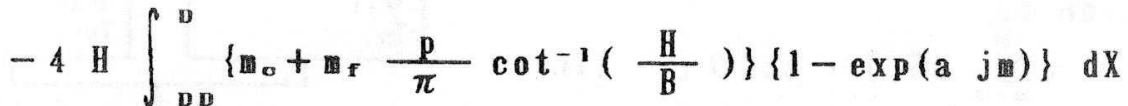

could be obtained.

If DD is larger than $D, j_{m}$ takes positive value for all over the main straight part of trackbelt. Tm could be calculated by substi-

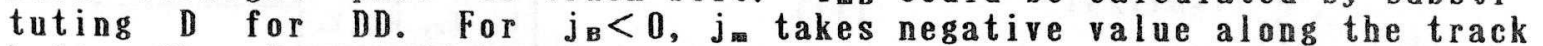
belt. Then $T_{m b}$ could be also calculated by substituting zero for $D D$.

The drag $T_{r b}$ and $T_{r b}$ developed on the contact parts of front and

rear whel could also be calculated as the integration of shear resistance. Therefore, the drag force $T_{3}$ is given by

$$
\mathrm{T}_{\mathbf{B}}=\mathrm{T}_{\mathrm{mb}}+\mathrm{T}_{\mathrm{fb}}+\mathrm{T}_{\mathrm{rb}}
$$

where $T_{r b}$ should be zero for the eccentricity es larger than $1 / 6$.

(b) Locosotion resistance

The vertical sinkage $S_{r}$ is also calculated as the suration of static sinkage given from bo $\left(1.2 p_{r}\right)^{b} \cdot \cos \theta_{e}$ and slip sinkage $S_{r i}$. For the inute interval d shown by

$$
d=-\frac{1}{M} \cdot i^{\prime} D \cdot \frac{D}{D-j_{B}\left(1+i_{b}\right)}
$$

, the vertical sinkage $S_{r i}$ could be calculated by use of Eq. (9) and (10). Then the locomotion resistance is given as the compaction resistance calculated from Eq. (11).

(c) Effective braking force

For each slip ratio ib from zero to - 100\%, the braking force $T$, and the effective braking $T_{4}$ could be calculated by use of the flow chart shown in Fig.3, and the results are shown in the same Fig. 4.

$T_{1}$ and $T_{4}$ decreases rapidly at smaller slip ratio $\left|i_{b}\right|$. Both $T_{1}$ and $T_{4}$ decreases monotonously at higher slip ratio $\left|i_{b}\right|$, where $T_{4}$ is usually saller than $T_{1}$ due to the increasing locomotion resistance. 


\subsection{Slip sinkage}

Fig. 5 shows the relations between $S_{r}, S_{r}$ and $i_{d}$ at driving state or $i_{b}$ at braking state. For both states, $S_{r}$ is always larger than $S_{P}$ due to the increasing slip sinkage with slip ratio. On the same slip ratio $i_{u}=-i_{b}, S_{r}$ at driving state is comparatively larger than that at braking state due to the difference of amount of slippage.

Fig. 6 shows the relation between $e_{s}$ and $i_{d}$ or $i_{b}$. At driving state, $e_{s}$ increases rapidly at higher value of slip ratio and reaches $1 / 6$ at $i_{d}=90 \%$ due to the large amount of slip sinkage $S_{r}$ At braking state, es increases gradually with the increment of $\left|i_{b}\right|$ and turns from negative value to positive one at $i_{b}=-56 \%$ due to the development of slip sinkage $S_{r}$. Fig.6 also shows the relation between $\theta_{t}$ and $i$ or $i_{b}$. At driving state, $\theta_{t}$ increases parabolically with the increment of $i d$. At braking state, $\theta_{\text {t }}$ increases almost linearly ith $\left|i_{b}\right|$.

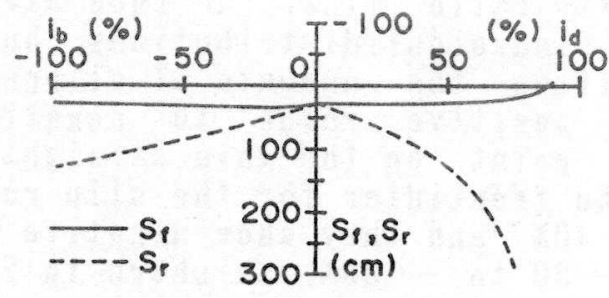

Fig. 5 Sinkage of front and rear whel $S_{F}, S_{r}$ and slip ratio $i_{d}, i_{b}$.

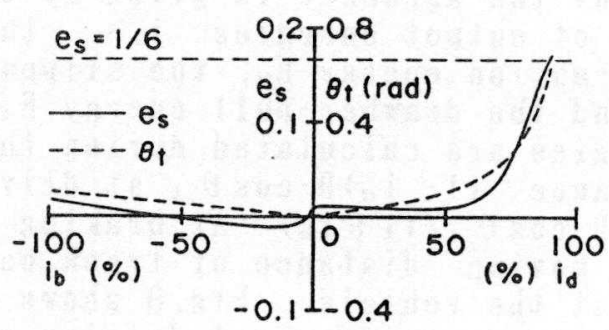

Fig. 6 Eccentricity es, trim angle $\theta_{t}$ and slip ratio $i_{d}$, $i_{b}$.

\subsection{Stress distribution}

At driving state, the amounts of slippage under track belt are positive for the hole range of the contact parts at any slip ratio. The normal stresses $\sigma$ and shear resistances $\tau$ under main straight part of track belt show positive sinusoidal distributions as shown in Fig. 7.

At braking state, the amounts of slippage change from positive value to negative one at some point on the main straight part of track belt
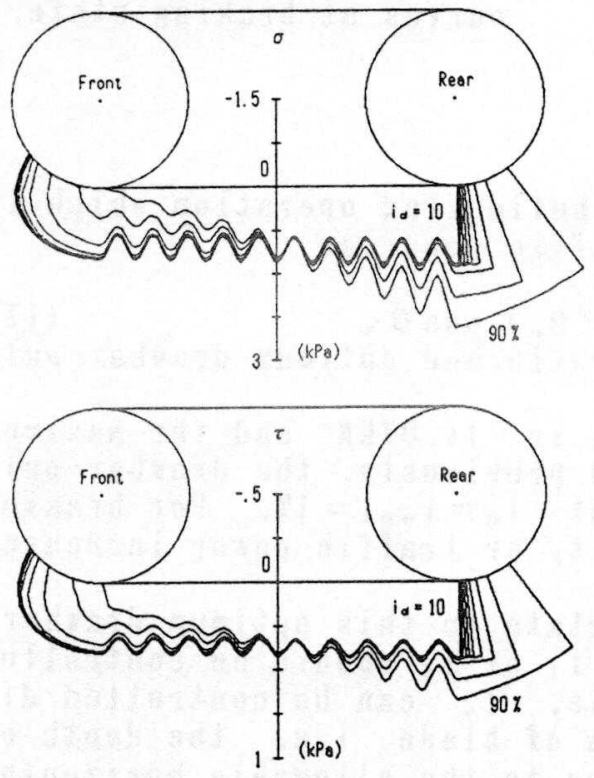

Fig. 7 Stress distributions at driving state.
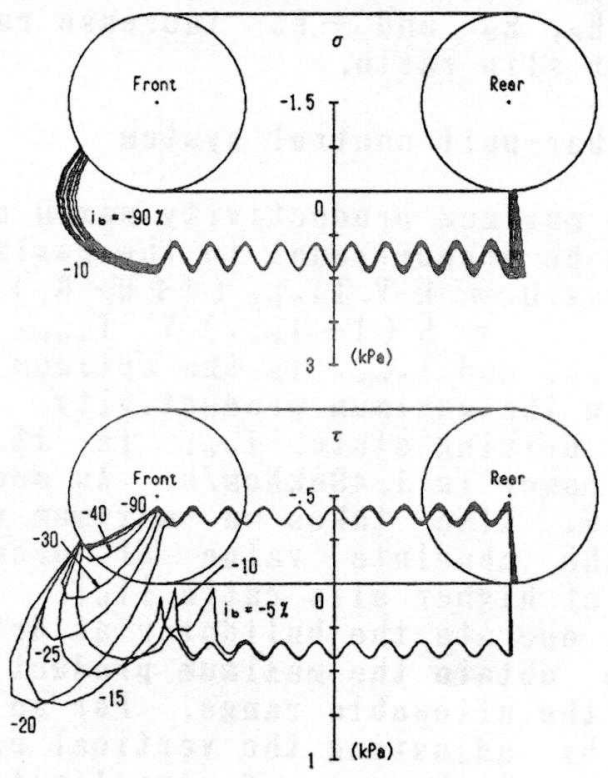

Fig. 8 Stress distributions at braking state. 
and on the front wheel with the increment of slip ratio $\left|i_{b}\right|$. o show always positive sinusoidal distributions, but $\tau$ ith resulting the amounts of slippage change from positive value to negative one at some point on the main straight part and on the frontidler for the slip ratio $i_{b}=0$ to $-40 \%$ and they show negative value for $\mathrm{i}_{\mathrm{b}}=-50$ to $-100 \%$, as shown in Fig. 8 .

\subsection{Energy balances}

The effective input energy $E_{1}$ supplied by the sprocket is given by the sumation of output energies i.e. the sinkage deformation energy $E_{z}$, the slippage energy $E_{3}$ and the drawbar-pull energy $E_{4}{ }^{4}$. Those energies are calculated during the running distance $\left(1-i_{d}\right) D \cdot \cos \theta_{t}$ at driving state or $D \cdot \cos \theta_{t} /\left(1+i_{b}\right)$ at braking state for the moving distance of track belt $D$ against the vehicle. Fig.9 shows the energy balance curves at driving state. E, decreases parabolically ith slip ratio from the maximum value $1.68 \times 10^{4} \mathrm{kNcm}$ at $i_{d}=3 \% . \quad E_{z}$ increases gradually ith slip ratio. Ea has some maximur value $6.49 \times$ $10^{3} \mathrm{kNcm}$ at $\mathrm{i}_{\mathrm{d}}=56 \%$ and reaches to zero at $i_{d}=83 \%$ where $T_{3} \cdot \cos \theta_{t}$ becomes to be $\mathrm{p} \cdot \sin \theta_{\mathrm{t}}$. $\mathrm{E}_{4}$ decreases almost linearly with slip ratio from the peak value $1.50 \times$ $10^{4} \mathrm{kNcm}$ at $\mathrm{i}_{\mathrm{u}}=1 \%$ and reaches to zero at $i_{d}=50 \%$. Fig. 10 shows the energy balance curves at braking state. These energies $E_{1}, E_{2}, E_{3}$ and $E_{4}$ develop remarkably in comparison ith those energies at driving state. $E_{2}, E_{3}$ and $-E_{4}$ increase rapidly at higher slip ratio.

\section{Drawbar-pull control syste日}

The raximum productivity rax.Q of the bulldozing operation which is given to be proportional to the raxinum trafic power as

$$
\begin{aligned}
\max Q & =k V T_{40 t}\left(1+H / R_{r}\right) \cos \theta_{t} \\
& =k\left(1-i_{\text {mot }}\right) V^{2} T_{40 t}\left(1+H / R_{r}\right) \cos \theta_{t}
\end{aligned}
$$

where iope and $\mathrm{T}_{4 \mathrm{p}}$ is the optimun slip ratio and optinum drabar-pull to obtain the maxirum productivity.

For driving state, iope is $1 \%, T_{4 u p t}$ is $14.07 \mathrm{kN}$ and the raximum traffic power is $1.498 \mathrm{kNc} / \mathrm{s}$. As mentioned previously, the drawbar-pull energy $E_{4}$ also takes a raxivur value at $i_{d}=i_{o p t}=1 \%$. For braking state, the absolute value of productivity or traffic power increases rapidly at higher slip ratio $\left|i_{b}\right|$.

To operate the bulldozer at driving state on this optimum drawarpull to obtain the maximum productivity, $T_{1}$ or ia should be controlled within the allowable range. For an exaple, $T_{4}$ can be controlled directly by adjusting the vertical position of blade i.e. the depth of excavation $t$ by use of some lirit sensors to the allowable horizontal excavation forces ${ }^{7)}$ given as

$$
\mathrm{T}_{4}=(1 \pm 0.2) \mathrm{T}_{40 \mathrm{pt}}
$$


$T_{4 a p s}=1.8 R_{B} B^{0} t^{2} 10^{-4.45 \alpha}$

where $B^{\prime}$ is the idth of blade. $R_{B}$ is the specific cutting resistance of soil and $\alpha$ is the rake angle.

\section{Conclusion}

To obtain the optimum operation of a bulldozer running on a super weak marine sediment, the optimu slip ratio should be determined to obtain the maximum productivity. To develop an automatically drawbar-pull control system, static and slip pressure sinkage curve and shear deformation curve on the interface between terrain and track belt should be determined firstly by use of each sensor. The relations between the driving or braking force, the drawar-pull or the effective braking force, and slip ratio could be determined rapidly from those terrain properties by use of the microcomputer simmulation programe. Afterwards, the optimum drawbar-pull and the optimum slip ratio could be auto matically determined for the given flexible tracked bulldozer. And the reasonable depth of excavation at the maximum productivity could be controlled by use of a depth sensor.

\section{References}

1) D. Rowland : Tracked Vehicle Ground Pressure and its Effect on Soft Ground Performance, Proceedings of the 4 th International Conference of ISTVS, Vol.1, pp.353-384, 1972.

2) T.Muro: Grouser Effect on Tractive Performance of a Bulldozer Running on a Superweak Marine Sediment, Proceedings of the 2nd AsiaPacific Conference of the ISTVS, pp. 355-366, 1988.

3) T.Muro: An Optimum Operation of a Bulldozer Running on a leak Terrain, Proceedings of the 5 th International Symposium on Robotics in Construction, JSCE, Vol.2, pp.717-726, 1988.

4) T.Muro, K. Omoto and A.Nagira : Traffic Performance of a Bulldozer Running on a leak Terrain Energy Analysis-, Proc. of ISCE, No. $403 / \mathrm{VI}-10,1989$.

5) M. Shimegi : Designing an Undervater Tractor, Proc. of the Conf. on Off-Highray Vehicle, Tractors and Equipments [GBR], pp.135-142, 1976.

6) T.Muro, K. Omoto and M. Futamura : Traffic Performance of a Bulldozer Running on a Weak Terrain Vehicle Model Test- Proc. of JSCE, No.397/VI-9, pp.151-157, 1988.

7) S.Hata : Construction Machinery, Kajima Press, pp.151-168, 1987. 